\title{
Familias, escuelas y estudiantes de educación superior
}

\section{Resumen}

Se presentan resultados de una investigación con estudiantes de educación superior, ideas respecto de su propia familia y la relación que ésta ha tenido con las escuelas a las que aquéllos han asistido. Existen múltiples trabajos acerca de la relación escuelas-familias en la educación básica, pero no en educación superior.

Si se considera estos dos ámbitos como los principales responsables de la socialización primaria, la aparente distancia que van tomando los estudiantes respecto de sus familias conforme se avanza en los niveles educativos, sólo se esperaría en términos de un desarrollo de la autonomía durante la juventud.

Los resultados obtenidos reflejan un "arropamiento" familiar importante y constante durante toda su trayectoria; además, se muestran respuestas de estudiantes universitarios y de tres Escuelas Normales, todos en Aguascalientes. En la mayoría de los casos pareciera que prevalecen familias tradicionales y estables que pueden ofrecer buenas condiciones para los estudios. 
Palabras clave: Estudiantes; educación superior; educación normal; familias; trayectorias escolares

\section{Introducción}

La mayoría de los países cuenta con sistemas educativos cuyas estructuras y formas de organización se desarrollaron durante el siglo XX; su finalidad principal ha sido la formación de los ciudadanos y el desarrollo integral de las personas. Esta idea aparece en los idearios educativos de los organismos internacionales, y actualmente forma parte del deber ser de la educación como un derecho humano fundamental (INEE, 2013); además, esta educación debe incluir como rasgos esenciales la inclusión, la equidad, la calidad, y estar dirigida hacia el desarrollo sostenible (PNUD, 2015).

Es interesante observar que el surgimiento de las instituciones educativas (así como las conocemos actualmente), no tiene más de uno o dos siglos y tienen una considerable similitud en diversos países; incluso, cuenta con mecanismos para poder normalizar la formación y hacer evaluaciones a gran escala con carácter comparable (UIS, 2013).

Es en este tiempo que las tareas de formación de los individuos pasaron del ámbito privado al terreno público (Angulo, 2016), de las familias a las escuelas, de ser parte de microculturas (Velázquez, 2007) a formar y transformar las macroculturas de los Estados-nación, y más recientemente, del mundo globalizado y virtualizado, de una sociedad mundial (Almaraz, 2012).

La organización de los sistemas educativos en el mundo se ha asociado de manera natural a procesos de desarrollo psicológico, a etapas evolutivas delimitadas por la edad (Berger, 2016; Trianes, 2012; Papalia, 2012; Rice, 1997). Así tenemos que, en el caso de México, para la infancia el tipo educativo es el preescolar y la primaria; para la adolescencia es la secundaria y el bachillerato; y para la juventud y primera adultez es la educación superior.

Diversos trabajos de investigación señalan que algunas condiciones familiares como el nivel socioeconómico y la escolaridad de los padres, entendidos por teóricos sociales como elementos del capital cultural, así como la participación de la familia 
en la escuela y su valoración favorable hacia los estudios, están directamente relacionadas con trayectorias escolares exitosas (García, Neira y Albert, 2016; Henderson y Mapp, 2002; Huerta, 2009; O'Shea, 2016; Redding, 2006; Rodríguez, 2014).

Pero más allá de reconocer la implicación de las familias en los procesos formativos escolarizados, es preciso comprender este fenómeno desde la voz de los estudiantes, pues esto permite conocer qué significa para ellos y cómo se concreta en determinadas prácticas.

Para ello, se desarrolló un proyecto de investigación de corte exploratorio en el que se realizó un primer acercamiento a las realidades familiares de un conjunto de estudiantes de educación superior a partir de un cuestionario estructurado con algunas preguntas básicas.

Reconocer cuáles son las características de los jóvenes que han llegado a la educación superior, especialmente aquellas que les brindan sus familias, y la forma en que éstas se han involucrado en su proceso formativo, constituye un paso importante en la generación de conocimientos que pueden estar en la base de la generación de políticas públicas orientadas a ampliar la cobertura, a combatir la deserción, a mejorar la calidad y a apoyar los procesos formativos de los jóvenes.

\section{Antecedentes}

Al igual que la familia, la escuela forma parte del desarrollo de los individuos, y ambas instituciones participan en su proceso de formación; por lo tanto, la evidencia científica da cuenta de esta relación desde aspectos escolares como los procesos de aprendizaje, el desarrollo de los estudiantes y los procesos de gestión escolar. Así, la participación familiar en la formación escolar -en cualquiera de estos aspectos- es considerada como una estrategia de éxito escolar (Loya, Arzola y Armendáriz, 2013).

El trabajo de Fernández, Álvarez, Woitschach, Suárez y Cuesta (2017) expone que hay una importante relación entre la implicación familiar y el rendimiento académico, revelando que la forma en la que los padres se involucran en la educación de sus hijos tiene efectos distintos en el aprovechamiento acadé- 
mico, dependiendo de cómo se entienda ese involucramiento, que puede ser desde ayudar con las tareas hasta tener una buena comunicación sobre los asuntos escolares. También afirman que, en la medida en que las intervenciones parentales promueven el desarrollo de la autonomía de los estudiantes, habrá mejores resultados académicos.

Debido a la relación que tiene el involucramiento familiar en el desarrollo integral del estudiante, diversos trabajos (Caspe, López y Wolos, 2007; Hernández, Gomariz, Parra y García, 2016; Krauss, Kornbluh y Zeldin, 2017) manifiestan los beneficios actitudinales, conductuales, de razonamiento moral, de desarrollo cognitivo-social y sentido de la justicia social que los estudiantes pueden lograr, cuando se establecen lazos de colaboración entre las familias y la escuela.

La Ley General de Educación (DOF, 1993) considera a los padres de familia en la educación básica, pues en su apartado VII se especifican las condiciones de participación de estos actores educativos y su grado de presencia en la gestión educativa en México. Para la educación superior, con la creación del Consejo Nacional de Participación Social en Educación (Conapase), se propuso la puesta en marcha de los Consejos Consultivos de vinculación para la Educación Media Superior y Superior, mismos que fueron impulsados en algunos estados del país, aunque sin mucho éxito (Canales, 2006).

Lo anterior expone que para la educación superior aún no hay normativa que disponga las condiciones de participación de las familias; sin embargo, en la realidad cotidiana de los estudiantes, los familiares continúan involucrándose en la vida académica de los universitarios, casi siempre a través de mecanismos informales, muchas veces de formas tangibles y otras de forma casi invisibles, pero altamente significativas para los jóvenes.

Cabe señalar que este trabajo se ubica en la línea de investigación que aborda la relación familias-escuela desde las posibilidades de desarrollo integral que este vínculo le pueda brindar al estudiante.

En el abordaje de la participación familiar en la universidad hay todavía más incertidumbres que certezas, y existe duda respecto a la pertinencia del estudio del tema a nivel universitario, 
puesto que los estudiantes son ya adultos que han alcanzado cierto grado de autonomía e independencia; por otro lado, no se descartan las afirmaciones de autores que señalan la importancia de mantener altos niveles de colaboración entre familia y escuela a lo largo de toda la trayectoria escolar, que en conjunto potencialicen las capacidades de los estudiantes (Comelas et al., 2013; Crosnoe, 2004; Oliva y Palacios, 2002).

El análisis de los rasgos culturales de las familias mexicanas supone que efectivamente brindan apoyo a los estudiantes, incluso en la universidad. El punto intermedio, como indica Solernou (2013), se encuentra en demostrar abiertamente la importancia de la presencia e influencia familiar para el estudiante universitario, sin caer en ninguno de los dos extremos: jóvenes totalmente desvinculados de sus familias o jóvenes dependientes y sobreprotegidos por éstas. O quizá lo mejor sería ubicarlos no de manera categórica sino de manera dinámica en todo este abanico de posibilidades.

La propuesta conceptual de Zayas, Corral y Lugo (2011) sobre definir en la educación superior involucramiento en lugar de participación, implica una connotación distinta a la de participación que se entiende en el nivel básico, pues este nuevo concepto se refiere al

conjunto de actividades voluntarias que los padres desarrollan [...] tanto en el hogar como en la escuela para influir en las prácticas y decisiones de los actores escolares de la educación superior en su conjunto [...] y con ello incidir en la formación escolar de sus hijos (p. 4).

Resalta la idea de actividad voluntaria, en estricto sentido, ya que como se dijo antes, en este nivel no hay normativa alguna que promueva u obligue la participación de las familias en los procesos formativos. Sin duda, es interesante la propuesta porque se desliga el significado de las actividades que realizan las familias en su paso por el nivel básico, aunque el concepto aún tiene la debilidad de que se remite únicamente a los padres y madres de familia como únicos actores que se involucran en la formación escolar de los estudiantes. 
En este trabajo también se recupera el concepto de involucramiento, no obstante, se piensa que en el caso de la educación media superior y superior, éste adquiere matices distintos y puede ser llevado a cabo por actores distintos a los padres, incluyendo a otros miembros de la familia como hermanos, abuelos, primos, tíos, y por lo tanto se habla ya de involucramiento familiar no sólo parental.

\section{Marco teórico}

Actualmente el concepto de familia ha mutado y en general se trabaja con un concepto abierto e incluyente, que abarca diversas estructuras, configuraciones y conformaciones, las cuales pueden o no estar "acreditadas" por las legislaciones como formas válidas, pero que existen socialmente tanto por convicción de sus integrantes como por lazos emocionales y afectivos, e incluso de conveniencia, y no necesariamente de sangre, como ocurría de manera tradicional.

La familia es una de las instituciones que cobra mayor importancia en el desarrollo de los individuos, y de una manera especial en los jóvenes, quienes por la etapa evolutiva que viven requieren soporte emocional y un acompañamiento que les permita la conformación de su identidad y el logro de la autonomía (Fajardo, Maestre, Felipe, León del Barco y Polo, 2017).

Así, la familia, en sus condiciones contextuales, y considerando el capital cultural con el que cuenta (evidenciado, por ejemplo, con un indicador como la escolaridad de los padres), su estructura, la dinámica que desarrollan sus miembros, el apoyo escolar que brinda a sus integrantes (el grado en el que los padres se involucran en las actividades escolares de los hijos) y las expectativas de aprendizaje que "heredan", pueden adquirir un papel importante en los procesos educativos como el rendimiento académico o la deserción.

Por ejemplo, en países latinos como México, Chile, Argentina y Perú el contexto sociofamiliar se asocia al abandono escolar en la educación media superior, de manera que los jóvenes que desertan no llegarán a la universidad y tampoco se insertarán a corto plazo en el mercado laboral (Díaz y Osuna, 2017). 
Sin duda, el nivel socioeconómico familiar puede influir en que los hijos asistan o no a la universidad; otro elemento que juega de manera importante son las expectativas familiares, pues el acceso a la educación superior está cargado de altas expectativas por parte de las familias, sin importar su condición económica, manifestándose en muchas de ellas el fenómeno de la primera generación en asistir a la universidad.

En este sentido, y de acuerdo con O'Shea (2016), el ingreso puede depender más bien de la percepción que los estudiantes tengan sobre sus propias capacidades. La autora plantea la clara influencia positiva que la familia tiene en el éxito académico de los estudiantes, pero que la relación no es solamente unidireccional, pues la universidad también influye en el incremento del capital cultural de la familia; así, la asistencia a la universidad proporciona un impacto (cognitivo, económico, de estatus social) en todos sus miembros, es decir, a nivel intergeneracional, y por ello las expectativas de la familia respecto del ingreso a la educación superior de sus miembros puede llegar a ser tan importante.

Durante la juventud, en los años ubicados entre la adolescencia (con sus cambios físicos y psicológicos) y la adultez (que supone una madurez y estabilidad tanto física como social), podemos encontrar la posibilidad de estudiar una carrera universitaria. Esto no quiere decir que sólo los jóvenes pueden estar en la educación superior, pero típicamente quienes cursan este nivel se encuentran entre los 18 y 25 años. Y esta condición etaria define en buena medida las lógicas en que funcionan las instituciones y las experiencias y significados que los estudiantes asignan a esta etapa formativa.

Goñi, Esnaola, Rodríguez y Camino (2015), al indagar con adolescentes, jóvenes y adultos las relaciones entre el autoconcepto personal y la satisfacción con la vida, poniendo a prueba un modelo estructural que contemplaba cuatro dimensiones del autoconcepto (autorrealización, honradez, emociones y autonomía), concluyen que el desarrollo de la responsabilidad individual y social a través de la educación, asociado directamente con el logro de una mayor autonomía, fomenta la satisfacción con la vida entre los estudiantes. 
Asimismo, Huéscar y Moreno (2017) realizaron una validación de la escala de "Apoyo a la autonomía del estudiante" con 332 estudiantes universitarios, y en uno de sus resultados señalan, a partir de un análisis de regresión lineal, que el estilo de apoyo a la autonomía predecía positivamente la satisfacción con la vida de los estudiantes de educación superior. Es decir, el paso por la educación superior no es sólo el tránsito por la juventud, sino que, si se trabaja de forma directa sobre el apoyo a la autonomía, se puede lograr mayor satisfacción con la vida, lo que puede indicar mejores procesos de desarrollo humano.

En este sentido, Madrigal (2010) afirma que las instituciones de educación superior deben asumir su responsabilidad social encomendada, y para ello deben implementar estrategias para formar en autonomía y en el pleno desarrollo del potencial humano de los estudiantes que se encuentran en los niveles de educación superior, enfatizando la formación de la autonomía como expresión de la libertad.

Desde esta visión, se vuelve una prioridad el estudio profundo de las familias desde su cotidianidad, en el que se identifiquen indicadores de convivencia, pautas de comunicación y conductas, así como prácticas que permean en las valoraciones que los hijos hacen hacia el estudio, que los llevan al (posible) acceso y culminación de su formación profesional, pues esta condición, más allá de brindar oportunidades de trabajo, genera posibilidades de desarrollo en los jóvenes.

\section{Metodología}

Al considerar la importancia del involucramiento familiar para los procesos formativos de sus integrantes, particularmente en el caso de quienes estudian en educación superior, y el poco conocimiento que se tiene de lo que pasa en este nivel educativo respecto a la relación familias-escuela, se diseñó una estrategia metodológica para conocer la opinión de algunos jóvenes a través de una encuesta, como parte de un trabajo exploratorio y transversal. El propósito fue conocer la percepción de algunos estudiantes de la Universidad Autónoma de Aguascalientes y de tres Escuelas Normales sobre el involucramiento familiar en su trayectoria escolar. 


\section{Participantes}

Se aplicaron cuestionarios a 465 estudiantes seleccionados de manera intencionada, sin buscar representatividad, a través de una red docente, quienes concedieron unos minutos de su clase para poder hacer la aplicación.

Las características generales de esta muestra fueron las siguientes: de los participantes, $75 \%$ fueron mujeres y $25 \%$ hombres; en cuanto al avance en su carrera, $38 \%$ eran estudiantes de primer semestre, $44 \%$ de tercero, y el resto de quinto, séptimo y noveno ( 7,9 y 2 respectivamente); por edades se obtuvo una distribución de 18 a 36 años, con una media de 20 años y desviación estándar de 2.5 años.

\section{Instrumento}

Se decidió trabajar con un cuestionario estructurado debido a que el tema abordado ha sido poco estudiado, para de esta manera realizar un acercamiento general y amplio que permita identificar futuras líneas de indagación; el instrumento fue de elaboración propia y consta de reactivos de opción múltiple, diferencial semántico, ítems tipo Likert y unas pocas preguntas abiertas.

Para su revisión y adecuación, se realizaron actividades de jueceo y piloteo; en el primer caso, se sometió a la revisión de dos profesores universitarios, investigadores, con experiencia en la temática de escuela-familia, y para la prueba piloto se aplicaron 20 cuestionarios a diferentes estudiantes, a quienes además se les preguntó sobre diversos aspectos de forma y contenido (claridad de las preguntas, la pertinencia y exhaustividad de las respuestas, la maqueta del instrumento).

A partir de las observaciones obtenidas de estos dos ejercicios, se realizaron mejoras y puntualizaciones en algunos reactivos, así como una reorganización en apartados y se redactaron las indicaciones buscando mayor precisión.

El instrumento está organizado en tres dimensiones: de identificación de los estudiantes (edad, género, semestre); información acerca de las familias, tales como estructura (la pregunta abierta fue "¿quiénes conforman tu familia?"), valoraciones acer- 
ca de la relación y la comunicación familiar, niveles de estudio de los padres, y condición económica familiar; así como situaciones escolares generales: promedio de calificación, y la valoración de la participación familiar actual en actividades escolares no sólo durante el tiempo de la educación superior, sino en toda la trayectoria educativa de los estudiantes. Para el análisis de la información se elaboró una base de datos con el programa estadístico SPSS, versión 19.

A continuación se presentan los hallazgos del estudio a partir de la percepción, valoración y experiencia de los propios estudiantes, acerca de cómo están organizadas sus familias y la cercanía o involucramiento que observan con respecto de la escuela, no sólo en la que están actualmente sino en las que han transitado. Conocer esta dinámica, a partir de la apreciación de los propios estudiantes, brinda elementos de análisis y discusión acerca de si la relación entre estos dos ámbitos es percibida como favorable para el desarrollo educativo (y psicológico) de los jóvenes estudiantes de educación superior.

\section{Resultados}

A partir de las respuestas al cuestionario, se identificó que los estudiantes encuestados consideran que tienen relaciones familiares con buenas condiciones generales, y que esta situación permanece durante toda la trayectoria escolar, pues reportan un alto grado de involucramiento en actividades de las escuelas en las que estudiaron los diferentes niveles educativos. También se observan algunas diferencias importantes dependiendo del tipo de institución de origen (universidad o escuela normal).

A pesar de las diferentes configuraciones familiares que reportaron (no sólo la familia nuclear tradicional, de padre, madre e hijos), un indicador que ofrece elementos para caracterizar el contexto de los estudiantes encuestados es el nivel de estudios alcanzado por los padres. Se observa que en ambos padres, $43 \%$ tienen educación básica (primaria y secundaria); la educación media es alcanzada por $32 \%$ de las madres y 26 $\%$ de los padres; y la educación superior (incluido el posgrado) representa $31 \%$ de los padres y $25 \%$ de las madres. 
Estos datos revelan que los padres y madres de estos jóvenes, en su conjunto, poseen un buen nivel de escolarización, pues $57 \%$ de ambos padres tiene educación media o superior; dicho de otra manera, más de la mitad de los padres o madres en esta muestra culminó el bachillerato o algo más, situación que resalta al comparar las cifras con otras entidades u otros grupos de este mismo grupo de edad.

A nivel nacional, el promedio de años de escolaridad de la población de 15 años y más es de sólo 9.2 años (apenas secundaria; hombres 9.3 y mujeres 9.0); y para el caso de Aguascalientes, es de 9.7 años (INEGI, 2015). Casi la mitad de los padres de esta muestra tendrían más de 12 años en promedio, e incluso algunos llegarían hasta 17 años o más (aquellos que tienen posgrado), lo que indica una diferencia sustantiva.

Por otro lado, la diferencia por género en el nivel de escolaridad alcanzado por los padres es más bien homogénea; con ello, podemos observar una condición estable y equilibrada en los hogares de los que provienen los estudiantes, pues ambos progenitores, en promedio, cuentan con una escolaridad similar, lo que seguramente se refleja en sus expectativas (favorables) hacia los estudios.

Si consideramos las diferencias por institución de origen, en la Tabla 1 vemos el siguiente contraste: un mayor porcentaje de padres de estudiantes universitarios alcanzaron la educación media y la superior.

Tabla 1.Escolaridad de los padres por tipo de institución (\%)

\begin{tabular}{lcc}
\hline & Estudiantes universitarios & Estudiantes de Normal \\
\hline Sin estudios & 0.3 & 2 \\
\hline Básica & 36 & 53 \\
\hline Media & 29 & 22 \\
\hline Superior & 35 & 23 \\
\hline
\end{tabular}

Una segunda condición que se exploró fue la valoración que los estudiantes hacen de su situación socioeconómica familiar; $61 \%$ menciona que cuenta con condiciones aproximadas de clase media (vivienda mediana con los servicios necesarios; 
vehículo familiar; asistir a eventos de entretenimiento pagado; acceso a la tecnología; usar la mitad del ingreso para las necesidades básicas), sólo 17 \% estaría en una situación de clase baja (vivienda propia o no pero con escasos servicios, pocas opciones de entretenimiento pagado, dificultad para adquirir tecnología e inversión de la mayoría del ingreso familiar en alimentos), y $22 \%$ de clase alta (vivienda propia y amplia con todos los servicios; dos o más automóviles; vacaciones regulares; renuevan constantemente la tecnología que usan; y el gasto del ingreso en alimentos es mínimo). En la Tabla 2 se muestran las diferencias por tipo de institución.

Tabla 2. Condición socioeconómica por tipo de institución (\%)

\begin{tabular}{lcc}
\hline & Estudiantes universitarios & Estudiantes de Normal \\
\hline Baja & 12 & 27 \\
\hline Media & 64 & 57 \\
\hline Alta & 25 & 16 \\
\hline
\end{tabular}

Esto puede indicar que las familias de este grupo de estudiantes tienen condiciones económicas aceptables en general y probablemente la oportunidad de estudiar en educación superior puede significar para estas personas la posibilidad de permanencia y/o movilidad social y económica.

Respecto de la estructura o configuración de las familias, casi tres de cada cuatro (72 \%) refieren que sus padres se encuentran "juntos", es decir, que están unidos ya sea por matrimonio civil o religioso, o por voluntad propia, y sólo 28 \% viven separados (no conviven, viudez o divorcio). Al igual que en los resultados anteriores, la Tabla 3 muestra las diferencias por tipo de institución.

Tabla 3. Estatus de los padres biológicos por tipo de institución (\%)

\begin{tabular}{lcc}
\hline & Estudiantes universitarios & Estudiantes de Normal \\
\hline Juntos & 68 & 78 \\
\hline Separados & 32 & 22 \\
\hline
\end{tabular}


Otro indicador que se utilizó en el cuestionario fue la opinión de los jóvenes acerca de la relación con su familia en general, utilizando una escala de 10 (Excelente) a 0 (Pésima): 82 $\%$ asignó los valores más altos, de 8 a 10, y sólo una persona señaló valores de 0 a 2 . Respecto de la comunicación al interior de la familia, los estudiantes encuestados consideran que es Buena (56 \%) o Excelente (13\%) y sólo siete personas (1.5 \%) dijeron que es Pésima.

Ante esto, pareciera que el conjunto de rasgos en las familias de los estudiantes cobra importancia respecto de la dinámica y las relaciones que se establecen entre los miembros, pues los jóvenes tienen una apreciación positiva de su familia en general.

En este sentido, y abonando a las ideas previas, en una tabla con indicadores en formato de diferencial semántico, utilizando emociones consideradas socialmente como opuestas (positivas y negativas; por ejemplo, felicidad y tristeza), señalan que cuando están con su familia se sienten aceptados (67\%), incluidos (63\%), y felices (62\%); cabe aclarar que estas cifras reportan sólo las respuestas para el valor 4, en una escala de 1 a 4, que en la codificación de este diferencial semántico es lo más cercano al sentimiento positivo.

Con estos elementos descritos (escolaridad, nivel socioeconómico, situación relacional de los padres y de la familia en conjunto) no es difícil pensar en estudiantes con buenas condiciones generales de vida, estables, incluso positivas, en las que pueden existir climas afectivos positivos, lo que en conjunto pudiera ofrecer a los hijos (estudiantes de educación superior, tanto universitarios como normalistas) los beneficios de un capital social y cultural amplio, o al menos el suficiente para afrontar esta etapa de su formación.

A partir del escenario propuesto hasta el momento, es necesario abordar la relación familias -escuelas a partir de lo que opinan los propios estudiantes. En general se observó que las familias, independientemente de los miembros y estructuras que las conforman, valoran mucho la escolarización y han tenido una participación cercana con las escuelas en las que han estado los estudiantes encuestados. 
Así, se encontró que sus familias tienen altas expectativas, pues los jóvenes expresan que sus estudios a nivel superior son muy importantes (71\%) o bastante importantes (21\%) para sus familias (ver las diferencias por tipo de institución en la Tabla 4), y consideran que la relación de ésta con las escuelas en las que han estado durante su trayectoria escolar ha sido buena (53 \%) o excelente (36 \%).

Asimismo, ante un reactivo de opinión que afirma "La relación entre la Universidad (o la Normal) y las familias debe ser muy cercana", casi siete de cada diez (72 \%) están de acuerdo o totalmente de acuerdo.

Tabla 4. Importancia de educación superior para la familia por tipo de institución (\%)

\begin{tabular}{lcc}
\hline & Estudiantes universitarios & Estudiantes de Normal \\
\hline Nada & 0.3 & 1 \\
\hline Poca & 2 & 1 \\
\hline Regular & 4 & 10 \\
\hline Bastante & 19 & 23 \\
\hline Mucha & 75 & 65 \\
\hline
\end{tabular}

Pareciera que la visión respecto de la escolaridad/formación/educación de los hijos no es algo reciente en estas familias, ni surgió ahora que tienen integrantes en una Institución de Educación Superior (IES). De acuerdo con la valoración de los propios estudiantes, a partir de una escala de 0 a 10 (de Nula participación a Muy activa), $91.5 \%$ afirmaron que en preescolar fue Muy activa (representa la suma de los valores 8 a 10), en primaria $89.4 \%$, secundaria $76.8 \%$, para bachillerato $65.1 \%$ y $63 \%$ para la educación superior.

Esto es, para cualquier tipo y nivel educativo, entre seis y nueve de cada diez estudiantes encuestados consideran que sus familias se han involucrado de forma muy activa en sus estudios durante su vida, lo que se ve reforzado con la valoración altamente positiva de parte de estas personas (estudiantes y sus familias) hacia la escuela en su conjunto. 


\section{Conclusiones}

En todos estos resultados se percibe que estudiantes como los de esta muestra son privilegiados, pues tienen acceso a la educación superior y además cuentan con buenas condiciones familiares que pueden sostenerles y apoyarles para el tránsito por este nivel educativo.

En los ideales expresados en todos los documentos normativos, acuerdos y leyes, se señala que la educación deberá provocar condiciones de igualdad social, bienestar general, y crecimiento armónico de las personas (entiéndase su desarrollo psicológico). Pareciera que la educación superior, con apoyo de las familias (ya sea formal o informal, institucionalizado o no), puede ofrecer alternativas de inclusión y lucha contra la desigualdad social y económica, lo que es observable en la alta valoración que muestran los estudiantes encuestados, y coincide por lo señalado por Silva y Rodríguez (2012) y Delgado, Martínez y González (2013) respecto del ingreso a la educación superior en general.

La realidad del sistema educativo, y de cada uno de sus subsistemas, es altamente compleja. Respecto de las relaciones que establecen las IES con las familias, se tiene la imagen de que los estudiantes, al ser considerados como mayores de edad, no requieren ni buscan la participación/inclusión/presencia de sus familias en esta etapa de su vida. Sin embargo, en los resultados de esta investigación se ha observado que los participantes expresan una situación muy diferente, en la que pareciera que las familias toman un papel protagónico, tanto real y objetivo como simbólico.

Al considerar la percepción de los estudiantes respecto del arropamiento que les ha brindado su familia, tanto en esta etapa de su vida académica, como a lo largo de su trayectoria escolar, aunque hay diferencias importantes entre los dos tipos de instituciones considerados, vemos que en ambos contextos las familias pueden ser propulsoras importantes para el desarrollo personal y psicológico de sus integrantes que participan de la educación superior, en su gran mayoría jóvenes de entre 18 y 23 años de edad. 
Como se mencionó antes, esta investigación tiene un propósito exploratorio. A partir de los resultados, y considerando los antecedentes presentados, surgen preguntas para nuevos acercamientos y posibles temas de investigación.

Por ejemplo, si se considera el trabajo de Goñi, Esnaola, Rodríguez y Camino (2015), acerca del autoconcepto personal y la satisfacción con la vida, o el estudio de Huéscar y Moreno (2017), podemos indagar en cuestiones como: ¿en qué medida las relaciones que establecen las familias con las IES favorecen o no la autonomía de los jóvenes? ¿Es necesaria la presencia de la familia en esta etapa de la vida o se debe propiciar una mayor independencia y distanciamiento? ¿Estos ámbitos, cada uno por separado y en conjunto, fomentan la satisfacción con la vida entre los estudiantes? ¿Qué significa cada una de estas situaciones para los estudiantes? ¿En qué grado requieren (o no) los procesos formativos en educación superior el involucramiento familiar?

También se mencionó antes que el concepto "familia" está cambiando; lo mismo pasa respecto a lo que significa cursar la educación superior. Si ambas instituciones sociales son tan importantes en el desarrollo de los individuos (Fajardo, Maestre, Felipe, León del Barco y Polo, 2017), ¿cómo afectan a los jóvenes los cambios que se producen actualmente respecto de ambos contextos en sus imaginarios, lógicas, posibilidades?

Será interesante continuar esta línea de investigación para indagar en la exploración y comprensión de las diferencias debidas a los tipos de institución, y discutir si los hallazgos se deben al entorno que ofrece la escuela, a mecanismos de reproducción social (condiciones familiares y personales), y en qué sentido podemos enfocar este conocimiento para la mejora de la educación superior y de los procesos de desarrollo personal de los jóvenes que por ahí transitan.

\section{Referencias}

Almaraz, José (2012). Hacia una formulación analítica de la sociedad mundial: de Rostow a Luhmann. En Papel Político, 17(2), 737-773. Recuperado de http://www.redalyc.org/articulo. oa?id=77727810012 
Angulo, Félix (2016). Lo público y lo privado en educación. En Revista Interuniversitaria de Formación del Profesorado, 30(1). Recuperado de http://www.redalyc.org/jatsRepo/274/27446519002/index.html

Berger, Kathleen (2016). Psicología del desarrollo: infancia y adolescencia. 9a edición. Madrid: Editorial Médica Panamericana.

Canales, S. A. (2006). La participación social en educación: un dilema por resolver. En Perfiles educativos, 28(113), 64-80.

Caspe, M., Lopez, M. y Wolos, C. (2007). Family involvement in elementary school children's education. Family involvement makes a difference: Evidence that family involvement promotes school success for every child of every age. Harvard Family Research Project. Number 2, Winter 2006/2007.

Comellas, M., Missio, M., Sánchez, L., Garcia, B., Bodner, N., Casals, I. y Lojo, M. (2013). Familia, escuela y comunidad: un encuentro necesario. Barcelona: Ediciones Octaedro.

Crosnoe, R. (2004). Social capital and the interplay of families and schools. En Journal of Marriage and Family, 66(2), 267-280.

Delgado, M., Martínez, C. y González, A. (2013). Familias y educación. En B. Salinas, Educación, desigualdad y alternativas de inclusión: la investigación educativa en México 2002-2011 (págs. 93-154). México: ANUIES, COMIE.

Diario Oficial de la Federación (DOF). (1993). Ley General de Educación. Última reforma publicada DOF 22-03-2017.

Díaz, K. y Osuna, C. (2017). Contexto sociofamiliar en jóvenes en situación de abandono escolar en educación media superior. En Perfiles Educativos, 39(58), 70-90.

Fajardo, F., Maestre, M., Felipe, E., León del Barco, B. y Polo, M. (2017). Análisis del rendimiento académico de los alumnos de educación Secundaria Obligatoria según las variables familiares. En Educación XX1, 20(1), 209-232.

Fernández, R., Álvarez, M., Woitschach, P., Suárez, J. y Cuesta, M. (2017). Parental involvement and academic performance: Less control and more communication. En Psicothema, 29(4). García, A., Neira, I. y Albert, C. (2016). Influencia del capital social y cultural en el conocimiento financiero de los adolescentes: entorno familiar y escolar. Ministerio de Educación.

Goñi, Eider, Igor Esnaola, Arantzazu Rodríguez y Igor Camino 
(2015). Personal self-concept and satisfaction with life in adolescence, youth and adulthood. En Psicothema, 27(1), 52-58, doi:10.7334/psicothema2014.105

Henderson, A. T. y Mapp, K. L. (2002). A new wave of evidence: The impact of school, family, and community connections on student achievement. Annual synthesis. National Center for Family and Community Connections with Schools. Austin: National Center for Family and Community Connections with Schools SEDL.

Hernández, M., Gomariz, M., Parra, J. y García, M. (2016). Familia, inmigración y comunicación con el centro escolar: Un estudio comparativo. En Educación XX1, 19(2), 127-151, doi: 10.5944/educXX1.14229.

Huerta, E. (2009). La relación escuela-padres en las secundarias mexicanas. Colección Cuadernos de Investigación. México: INEE.

Huéscar, Elisa y Antonio Moreno (2017). Apoyo a la autonomía entre estudiantes, estrés percibido y miedo a la evaluación negativa: relaciones con la satisfacción con la vida. En Behavioral Psychology / Psicologia Conductual, 25(3), 517-528.

Instituto de Estadística de la UNESCO (UIS). (2013). Clasificación Internacional Normalizada de la Educación. CINE 2011. Montréal: UNESCO-UIS.

Instituto Nacional para la Evaluación de la Educación (INEE) (2013). El derecho a la educación en México. $2^{\mathrm{a}}$ edición. México: INEE. Recuperado de http://publicaciones.inee.edu.mx/detallePub.action?clave=P1D218

Krauss, S., Kornbluh, M. y Zeldin, S. (2017). Community predictors of school engagement: The role of families and youth-adult partnership in Malaysia. En Children and Youth Services Review, 73, 328-337.

Loya, C. y Arzola, D., y Armendáriz, H. (2013). La participación de los padres de familia en el ámbito de la RIEB. En IE Revista de Investigación Educativa de la REDIECH, 4(7), 33-42.

Madrigal, Arturo (2010). Formar en autonomía: una visión desde Beck para la educación superior. En Revista Politécnica, 6(11), 18-24. O'Shea, S. (2016). Avoiding the manufacture of "sameness": first-in-family students, cultural capital and the higher educa- 
tion environment. En Higher Education, 72(1), 59-78.

Oliva, A. y Palacios, J. (2002). Familia y Escuela: padres y profesores. En Rodrigo, M. y Palacios, J. (coords.), Familia y desarroIlo humano. Madrid: Alianza Editorial.

Papalia, Diane (2012). Desarrollo humano. 12ª edición. México: McGraw Hill.

Programa de las Naciones Unidad para el Desarrollo (PNUD) (2015). Folleto sobre los Objetivos de Desarrollo Sostenible. Recuperado de http://www.undp.org/content/undp/es/home/ sustainable-development-goals.html

Redding, S. (2006). Familias y centros escolares. México: CENEVAL, DIE-CINVESTAV, COMIE, INEE y UPN.

Rice, Philip (1997). Desarrollo humano: estudio del ciclo vital. $2^{\mathrm{a}}$ edición. México: Prentice-Hall Hispanoamericana.

Rodríguez, E. (2014). La influencia de los factores familiares en el rendimiento académico. Tesis de grado. Valladolid: Universidad de Valladolid.

Silva, M., y A. Rodríguez (2012). El primer año universitario entre jóvenes provenientes de sectores de pobreza: un asunto de equidad. México: ANUIES.

Solernou, I. (2013). La familia y su participación en la universalización de la educación superior. En Educación Médica Superior, 27(1), 54-63.

Trianes, Victoria (2012). Psicología del desarrollo y de la educación. Madrid: Ediciones Pirámide.

Velázquez, Luz (2007). Microculturas y trayectorias estudiantiles en el nivel medio superior. En Tiempo de Educar, 8(15), 37-65. Recuperado de http://www.redalyc.org/articulo.oa?id=31181503

Zayas, P., Corral, G. y Lugo, M. (2011). El involucramiento de padres y madres de familia en la educación superior. En Memoria electrónica del XI Congreso Mexicano de Investigación Educativa. México: COMIE. 